\title{
New Approaches in Philips ECG Database Management System Design
}

\author{
S Zhou, G Guillemette, R Antinoro, F Fulton \\ Diagnostic ECG Group, Philips Medical Systems, \\ Oxnard, California, USA
}

\begin{abstract}
Electrocardiogram (ECG) databases play an important role in medical research, pharmaceutical research, medical education and health care. Due to growing demands in research, training, and health care, designing and managing such ECG databases has become a complex problem. This paper reports on the new design approach and the new application model of the Philips ECG Management System (EMS). The Philips EMS is designed not only to store and manage ECG data, but also to automate the ECG workflow, to facilitate the ECG editing and confirmation process, to compare the serial ECG recordings, to capture and store auditing information, and report the serial ECG changes, and most importantly, to communicate with other systems, such as hospital information systems etc. The patient demographic and clinical data and ECGs are managed by a relational database. The system allows authorized users to access ECG data, ECG measurements and ECG interpretation in data format that is written in eXtensible Markup Language (XML), and to retrieve clinical cases for research and education by simple structured query language queries. In summary, the Philips EMS provides a powerful and easy-to-use tool to support research, education, and ultimately, to enhance patient care.
\end{abstract}

\section{Introduction}

Medical research, medical education and patient care require ECG databases and database management systems [1, 2]. Numerous research institutions, healthcare organizations and manufacturers have designed and developed such database management systems over the last few decades, and only a small portion of systems achieved success $[3,4,5]$. The main challenge is due to rapid technology evolution. The current "state-of-art" technology often becomes obsolete in a couple of years if not by months. Designing such a sophisticated ECG database management system in an era of rapid evolution of clinical and information technology has become increasingly complex. Sizeable resources are consumed in development and maintenance. As part of Philips Medical Systems' longstanding commitment to the medical community, substantial efforts have been made continuously to the ECG management system. This paper is to report the new design approaches of Philips ECG Management System with a focus on its flexibility and openness in data access.

\section{Technical methods}

The new design approaches adopted in the current Philips ECG Management System include open industry standards for data storage, convenience in user access and data query, communication, security, configurable workflow, auditing and tracking.

\subsection{System architecture}

The Philips EMS design is based on industry standard three-tier architecture and divided into three major components including the underlying layer of relational database, the upper layer applications for ECG viewing, editing, data retrieval and data aggregation, and the middle layer to adapt a flexibly configurable workflow according to the practices of individual institutions (Fig.1).

\subsection{ECG XML format}

ECGs stored in the system are in industry standard XML format. The most significant step Philips Medical System has taken is to make the XML data format 
schema available to the users. This new approach provides the users with maximum access to the data stored and supports the system interoperability.

\subsection{Relational database}

Structured Query Language (SQL) server is the core of database management in this system. The Philips EMS database is composed of normalized tables containing patient demographics, digital ECG waveform, measurements and ECG interpretation. Essentially, most ECG data are exposed via individual columns in relational tables [6]. This design enables the use of simple to advanced SQL queries for information retrieval [7].

\subsection{Secured user access}

Secure sockets layer (SSL) is supported to provide encrypted hypertext markup language (HTML) protocol whereby anywhere, anytime access can be provided to authorized users. Secure web-based application for ECG viewing and printing from the system is supported over the intranet and internet. Remote ECG editing is supported over the internet and Microsoft (dot).Net web-based editor whereby ECGs can be reviewed and confirmed by clinicians at their convenience.

\subsection{Web server}

Bidirectional information relay is performed by an industry standard web server. The server can relay user requests via web-based application to the back-end database layer, or forwards the database information resulted from the backend database to the web-based application.

\subsection{Workflow}

The workflow layer is configurable to meet the needs of a wide variety of practices, including automated pre-processing such as routing of incoming new ECGs for review via hard copy reports, diagnostic serial ECG comparison, notification via email, automated in-box assignment, additional special processing on confirmation of ECGs, including exporting the ECG in a variety of formats, such as XML [8], and Health Level Seven (HL7) to a variety of external systems, including HIS billing, order management and enterprise clinical information systems.

\section{Three-tier System Architecture}

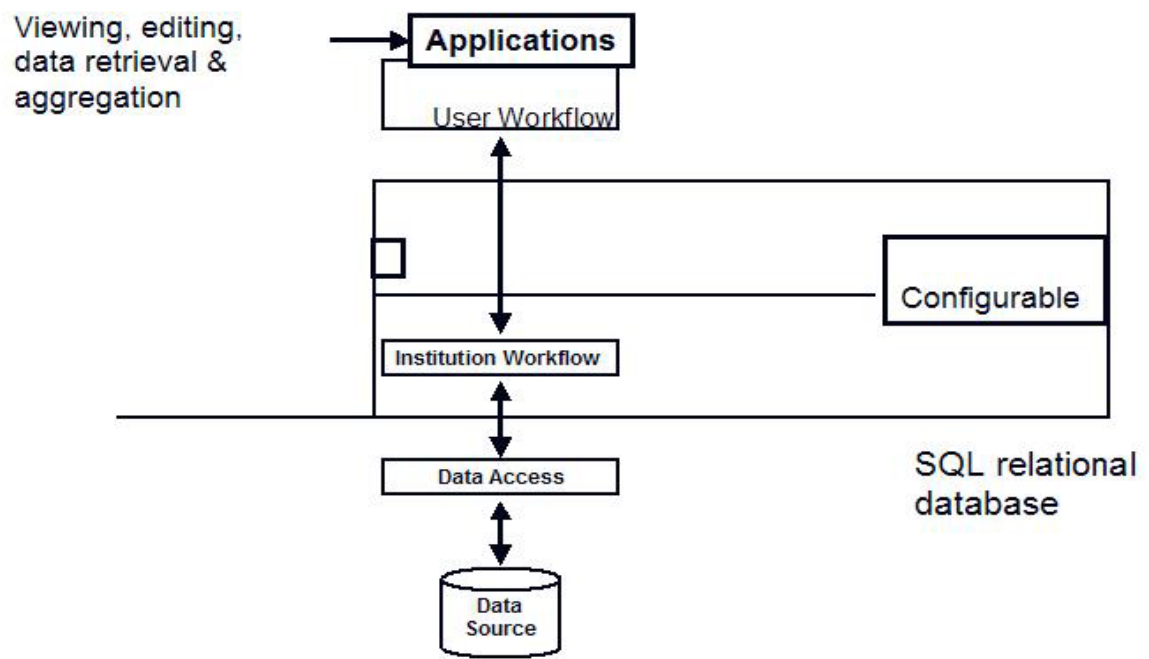

Figure 1. The three-tier client/server system architecture is divided into the lower-tier relational database, the mid-tier workflow and the upper-tier application as shown above. 


\subsection{Auditing and tracking}

The system is designed with auditing and tracking capability, and supports full auditing and tracking of ECG access and the operations and actions performed on individual ECGs. This capability facilitates the variable needs of users in different settings to comply with regulatory requirements.

\subsection{System communication}

A rich set of communications protocols are supported, including TCP/IP over local area network (LAN) and wide area network (WAN), and transmission control protocol/point-to-point protocol (TCP/PPP) over modem and dialup connections for communication with Electrocardiographs for transmission of ECGs to the Philips EMS for storage and subsequent review. Bidirectional communications between the EMS and the hospital information system (HIS) is supported using Unicode [9] encoded XML and HL7 message exchange (Fig.2). Exchange typically consists of importing ECG orders from HIS Data Order Entry system, exporting
ECG order fulfillment to the HIS billing system and confirmed ECG readings to the enterprise clinical information system.

\section{Applications}

Application models are designed with focuses on medical research, medical education and patient care. Several new application models have been adopted in the latest design.

\subsection{Medical and pharmaceutical research}

Ownership of the ECG database on a commercial management system has been debated over a few decades. The openness of the industry standard XML format, a major breakthrough design approach in the Philips EMS, allows researchers to maintain ownership of the ECG database and enable access with ease. Issues related to propritary ECG format no longer exist. One major effort made in the system design is to allow easy access for researchers, cardiologists, and healthcare

\section{System Communications}

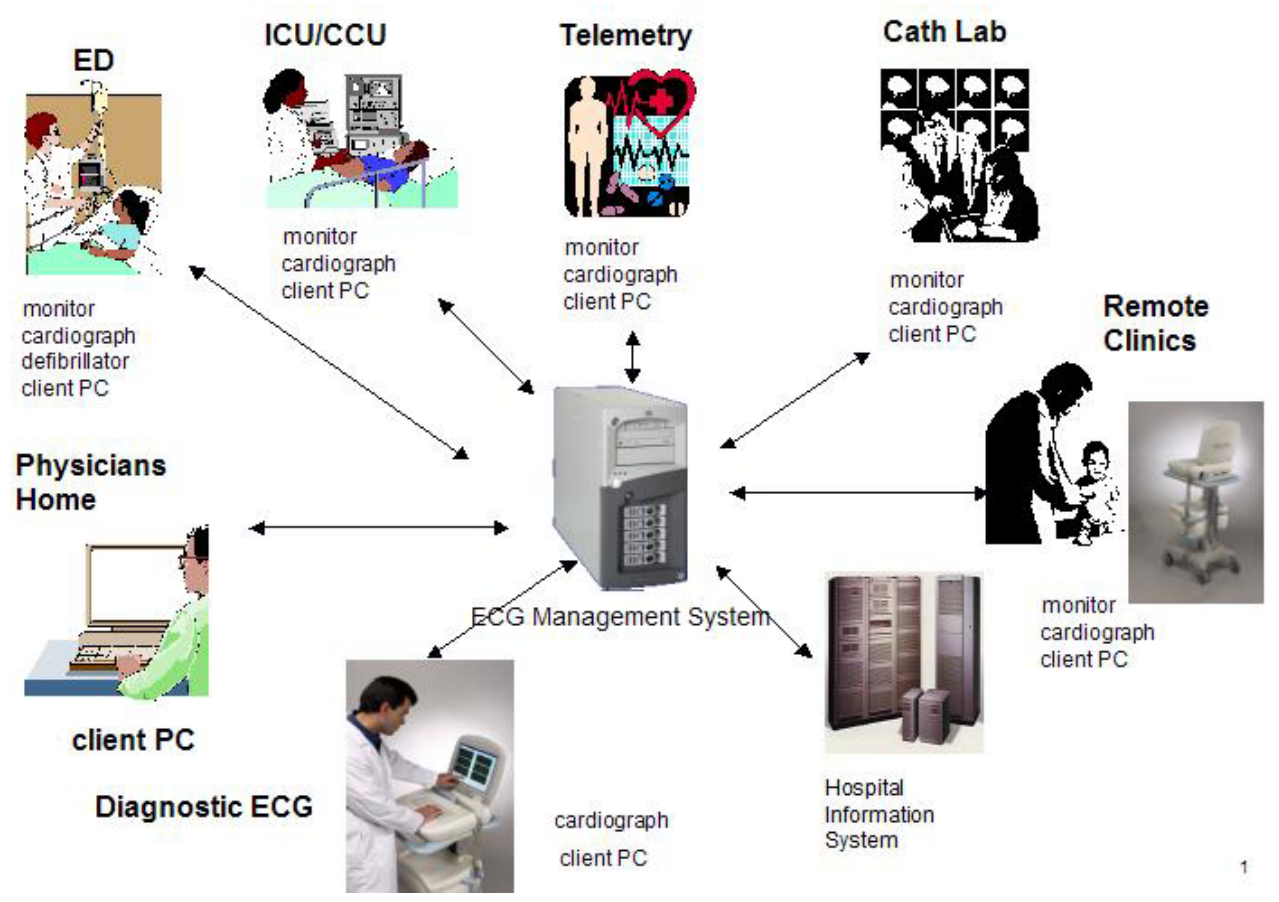

Figure.2. Communication between the ECG management system and the ECG devices is depicted in this diagram. Bidirectional communication requirements increase the complexity significantly. 
professionals. Researchers in medical and pharmaceutical studies have complete flexibility in data storage and retrieval, and in accessing and searching ECG by measurements, interpretation or the high fidelity digital waveform.

\subsection{Medical education}

ECG reading is an art in medical science. However, ECG examples shown in medical school textbooks are very limited. Without an ECG database management system, training ECG reading can be difficult. In the Philips EMS, ECG examples can be searched, retrieved, displayed and printed in seconds. The database can be queried easily using an SQL editor and tools. Essentially all ECG data is exposed via individual columns in relational tables. Using SQL's powerful commands such as "SELECT and JOIN", virtually any search query can be submitted to the system, and results can be obtained quickly.

\subsection{ECG management in patient care}

Most hospitals in the United States have experienced a shortage of experienced ECG professionals in recent years. The Philips EMS automates ECG order handling and reporting of order results to offset the resource shortage. The flexible workflow model and order handling capability work together to maximize process automation - from downloading ECG orders from HIS order entry system, uploading ECG order fulfillment data to the HIS billing system and sending ECG confirmed results to the enterprise clinical information system. All of the above facilitates handling of ECG data and aid decision-making for patient care. Another advanced application among many on the ECG management system is to perform serial ECG comparisons $[10,11,12]$. Serial comparison algorithm assists cardiologists in ECG diagnosis and evaluating progression and regression of a cardiac condition.

\section{Summary}

In summary, the system design is focused on the needs of researchers and health care professionals. The design is based on open industry standards and a networked three-tier client/server model. The system is adaptable and scalable to a wide variety of United States and international IT environments and requirements [13]. The ECG data stored is completely exposed via relational tables. The system meets and satisfies a variety of business and clinical information requirements. Although the system is non-trivial and may be considered a complex clinical information system, it is easily accessible via a standard web-based application, it is highly configurable to a wide variety of requirements in diagnostic cardiology departments for enhanced patient care, and its ad-hoc SQL querying capability makes it easy to use but powerful.

\section{Acknowledgements}

The authors acknowledge Alan Risse, Gary Okumura, Leigh Wells, George Diller, Krystyna Niklarz for their contribution in the new design of Philips ECG Management System.

\section{References}

[1] Gamon R, Cooper A: Applying an ECG database to aid decision-making in the A\&E. Accid Emerg Nurs 2002;10(2):62-62.

[2] Norman JE, Bailey JJ, Berson AS, et al.: NHLBI workshop on the utilization of ECG databases: preservation and use of existing ECG databases and development of future resources. $\mathrm{J}$ Electrocardiol, 1998;31(2):83-89.

[3] Chiarugi F, Lombardi D, Lees PJ, et al.: Support of daily ECG procedures in a cardiology department via the integration of an existing clinical database and a commercial ECG management system. Ann Noninvasive Electrocardiol, 2002;7(3):263-270.

[4] Nose Y, Akazawa K, Yokota M, et al.: An electrocardiogram database incorporated into the hospital information system. Med Inform (Lond), 1987;12(1):1-9.

[5] Wang C, Ohe K, Sakurai T, Nagase T, Kaihara S: An ECG storage and retrieval system embedded in client server HIS utilizing object-oriented DB. J Med Syst 1996;20(10):35-43.

[6] Codd EF: The Relational Model for Database Management: Version 2, Addison-Wesley, 2002.

[7] Date CJ: An Introduction to Database Systems. $8^{\text {th }}$ edition, Addison-Wesley, 2003.

[8] Goldfarb CF, Prescod P: XML Handbook. Prentice Hall, $4^{\text {th }}$ edition, 2001.

[9] The Unicode Consortium: The Unicode Standard, Version 3.0. Addison-Wesley Longman, 2000.

[10] Ariet M, Crevasse L, Caverly S, Leggett S, Greenfield J: Computerized serial comparison of electrocardiograms. J Electrocardiol 1991;23(supp):123-131.

[11] Crevasse L, Ariet M: Clinical usefulness of computerized ECG systems. J Med Syst 1987;11(1):21-24.

[12] Hedstrom K, Macfarlane PW: Development of a new approach to serial analysis. The manufactuer's viewpoint. J Electrocardiol 1996;29(Supp):35-40.

[13] Philips Medical Systems: TraceMaster ECG Management System User's Guide. U.S.A., 2003.

Address for correspondence.

Sophia Zhou PhD, FACC

Philips Medical Systems

1201 N Rice Ave., Oxnard CA USA 93030

Sophia.zhou@philips.com 